\title{
The system of chemical and mathematical problems as a tool for the development of motivation of students of technical universities
}

\author{
Galiya Igtisamova*1, Zemfira Yangirova ${ }^{1}$, Doniyor Nosirov ${ }^{1}$, and Aydar Yangirov ${ }^{2}$ \\ ${ }^{1}$ Ufa State Petroleum Technological University, Branch of the University in the City of Oktyabrsky, 54a, Devonskaya St., \\ Oktyabrsky, Republic of Bashkortostan, 452607, Russia \\ ${ }^{2}$ Institute of law of Bashkir State University, 131, Dostoevskogo St., Ufa, Republic of Bashkortostan, 450005, Russia
}

\begin{abstract}
The research relevance is connected with the orientation of the modern model of education towards the formation of a competitive creative personality, with the need for constant self-improvement and self-development. It should be noted that the effectiveness of student learning is largely determined by the level of motivation, interest and personal participation of subjects. Therefore, the article is aimed at disclosing mechanisms for the development of students' learning motivation through a system of differentiated tasks. The leading approach to study of a problem was a personal approach, the purpose of which is to identify the issue under discussion from the point of view of dialogism, subjectivity and individuality. This document presents the characteristics of the developed and tested system of differentiated problems of mathematics, focused on the development of student learning motivation, as well as the provision of empirical data on the results of implementation. It also describes the principles on which we defined three levels of tasks in mathematics: "algorithms", "tasks of finding tasks" and "creative tasks".
\end{abstract}

\section{Introduction}

The relevance of the problem of developing learning motivation for students in technical universities is based on a number of reasons. First, it should be noted that an insufficient level of school education and a lack of selforganization and self-reflection skills can cause an unfavorable psychological situation [1] and, therefore, become a "barrier" mechanism for studying chemical and mathematical disciplines. [2] Secondly, the high degree of abstraction of the material under study and its poor orientation towards future professional activities lead to a decrease in interest and a corresponding increase in students' anxiety. Thirdly, a high level of motivation for learning is a key factor in the preparation of future highly qualified specialists of the highest class. The motivation of learning is characterized by value orientations [3], motives and interests aimed at studying disciplines, shows the willingness of students to independently determine the goals of their activities, to achieve their aspirations, as well as to confirm their knowledge, skills and abilities.

\section{Goal of research}

The goal of our research is to study the motivation of students, as well as factors and pedagogical relationships that affect the motivation of students of technical universities. Given the development of learning motivation in higher education, we consider it reasonable to characterize students as a special social category. Student years are a period of intensive socialization, the development of higher mental functions, the formation of the entire intellectual system and the personality as a whole. At the same time, this is the age of a student who has a number of contradictions that it is advisable to take into account in the learning process between the heyday of intellectual and physical strength and the limited amount of time, the economic potential to meet the increased needs; between the desire for independence in the choice of knowledge and methods and forms of education; between the volume of information and the unwillingness to systematize and use it (overcoming these contradictions becomes possible taking into account the psychophysiological and individual personal characteristics). This approach ensures the creation of a "success situation" for each student, the conditions for the implementation of the "importance of disciplines" in the context of future professional activities. It should be noted that the "success situation" is a purposeful and organized combination of conditions under which the possibility of achieving significant results in its activities is realized.

\section{Results and discussions}

Psychological and pedagogical researches noted that the "success situation" contributes to the development of cognitive interest, corrects individual traits (anxiety, insecurity, self-esteem), supports a favorable

Corresponding author: Igtisamova_galiy@mail.ru 
psychological climate and significantly increases the motivation of learning [4]. It should also be noted that motivation is the leading factor that regulates the activity and behavior of a person. This position is confirmed by Yerkes-Dodson's law, which illustrates the dependence of the result of activity on the degree of motivation.

The analysis of psychological, pedagogical and methodical literature, as well as the study of the experience of other scientists in the development of the problem under discussion, showed that the problem of developing the motivation of studying students in educational mathematics [5] should be clarified and developed. This is primarily due to a change in the educational paradigm, methods of updating and requirements for the level of training of graduates, etc.

The above discussion allowed us to formulate a research hypothesis: the development of students' learning motivation in teaching mathematics and chemistry will be more effective if a system of differentiated tasks is developed and implemented [6]. A task can be considered as a simulated problem situation associated with a person in a cognitive process. It should be noted that the task of training future engineers are of key importance. This is due to the fact that, firstly, it ensures the integrity, adaptability and consistency of the educational process. Secondly, in the process of solving problems, students apply knowledge and experience, acquire skills in analysis, modeling and forecasting. Applied problems are essential to the educational practice of engineering students.

The didactic potential of applied problems is due to its ability to emphasize the importance of and develop an understanding of the value of mathematics and chemistry in the general training of engineers.

In addition to the formal knowledge of formulas, definitions and laws, the student must be able to put into practice the techniques of logical reasoning and evidence. The use of such tasks is very important, because, in addition to the formation of certain skills, it helps to create a success situation and to reduce the level of anxiety of first and second-year students. "Problemresearch tasks" are aimed at the development of analytical and research abilities, skills of modeling processes in living and inanimate nature, interpretation and data evaluation (tasks of reproductive level). Tasks at this level are important because the research process involves a complex of three successive stages: modeling, direct research and interpretation. These stages relate to the types of professional activity of the tolerant [7] future engineer.

Simulation is the construction of a mathematical model of the real situation by transferring parametric [8] problems to the language of mathematics. At this stage, students learn to analyze the situation, highlighting the essential and secondary relationships between the data, determining their completeness, and describing it using mathematical language. The second stage includes the study of a mathematically constructed model and means of scientific disciplines. Here, students will learn to find the most suitable method for solving a problem, choose methods and a sequence of actions, use mathematical tools, differentiating complex tasks and simpler subtasks.
Interpretation involves correlating the results with the original data, in other words; transfer of the answer and its assessment in accordance with the specifics of their future professional activity. At this stage, students learn to draw conclusions on solving problems, conduct a qualitative analysis of the data, and to establish causal relationships of the results. As a rule, problems of this type are solved by students with more sustained motivation and a fairly high level of awareness of the importance of mathematical disciplines in their future professional activity. Creative tasks include open type tasks or partially open type tasks. Open type tasks have a "blurry" condition, from which it is not clear how to act. These tasks require different solutions, contribute to the formation of research and reflexive skills, the ability to predict. Such tasks play an important role in training future engineers for the reason that they allow to realize the idea of a heuristic approach to solving a problem, use non-standard thinking, etc. As a rule, creative tasks are chosen by students with a high level of motivation, selfcontrol and self-assessment. They demonstrate fluency in the chemical-mathematical apparatus and knowledge of sciences, they are able to offer non-standard solutions to the situation, as well as interpret data and predict possible consequences. For a more detailed understanding of this idea, we give examples of all types of tasks from mathematics courses: differential equations. It should be noted that in the future, the training of engineers on this topic occupies a leading position. This is due to the fact that having arisen from problems of an applied nature, differential equations were reflected in many sciences after the further development of the theory. Therefore, the study of this topic contributes to the implementation of interdisciplinary connections. Secondly, in the process of learning the basics of the theory of differential equations, the student gains invaluable experience in real process modeling.

These functions were implemented to solve problems at all levels of complexity.

Example 1. Algorithm: Solve the equation $y^{\prime \prime}-y=e^{x}\left(x^{2}-1\right)$.

In the process of solving the problem, students should know the basic concepts, facts and methods of the topic "Differential Equations"; be able to solve differential equations and systems of differential equations of the simplest types, and to know the methods of the theory of differential equations and mathematical modeling. [7]

Example 2. How many isomeric homologues of benzene can correspond to the formula $\mathrm{C}_{9} \mathrm{H}_{12}$ ?

It should be borne in mind that this type of tasks can form an idea of the connection of methods for solving differential equations with mathematical models of chemical processes, as well as a valuable idea of methods for solving differential equations for a future professional, thereby contributing to the interests of students in the study of mathematics and natural sciences.

Experimental testing was conducted on the basis of the higher educational institution "Branch FSBEI HE 
USPTU in the city of Octyabrsky." The study involved 29 people. For the experiment, 2 groups were selected: the control (CC) and experimental (EE) groups. Experimental testing included three consecutive stages: preparatory, main and final.

At the preparatory stage, tasks related to the development of information and methodological support were implemented based on the analysis of the university's work programs.

Analysis of the psychological and educational literature, taking into account the above-listed mathematical educational functions (cognitive and applied), determined the reasonableness of the implementation of the following principles: fundamental, practical orientation, informatization, planting of greenery and saving.

The fundamentality principle is the key to the formation of a methodological culture of a professional and is to ensure the consistency and scientific nature of the preparation process. The practical orientation principle is aimed at solving the problem of matching a future career with the actual content of training. It is appropriate to pay attention to the ongoing scientific discussions: the fundamental is the main priority, which is based on theoretical foundations and practical orientation (applicable nature of engineering education).

The next organization principle and implementation of the mathematical foundation of engineering students is the informatization principle. This is caused by two main aspects. The first of these is determined by the conditions of the modern information society, characterized by a continuous increase in the information volume, a high level of information culture of competitive specialists, and the satisfaction of information needs using modern information and communication technologies. The second aspect is directly related to the intellectual component of technology, which includes the ability to work with information and to systematize it, analyze, synthesize and generalize.

The planting of greenery principle provides an environmental focus for training future engineers. This is due to the fact that a modern engineer must not only conduct research, predict and model technical objects, but also assess the environmental risks of his activities. The planting of greenery principle involves the consideration of the content of education in terms of the analysis, assessment, and prediction of environmental safety of nature. Research on the environmental component of training future engineers emphasizes that it is necessary to have not only a safety culture but also risk-oriented thinking. This approach is due to the fact that conservation issues are considered as the most important priorities in the professional activity of an engineer.

The above principles are reflected in the organization of the preparatory phase of the study. At this stage, students become familiar with the principles of working with the tasks system and with the system for evaluating results learning outcomes.

To conducted setting and justification of the study objectives, as well as the development of students' cognitive interest in the chemical and mathematical disciplines and future profession. In addition, at this stage, the diagnostics of the initial level of student learning is carried out, and the requirements for the level of mastering professional knowledge and the competence formation are established.

The teacher performs organizational, control, diagnostic functions; acquaints students with the course program, technology of information search and processing, requirements for conducting written examinations in independent study, contributes to the formation of an individual educational direction implemented on the main stage.

The main stage includes the assimilation of educational content, represented by a set of theoretical and practical differentiated tasks. This step allows the teacher to manage the training process, coordinate the independent work of students with students, to make adjustments in accordance with the individual course of study, ensuring the continuity of the learning process. This allows you to organize the effective work of students with educational materials and implement them in accordance with established deadlines.

At the stage of evaluation and reflection, knowledge and its application in practice, the implementation of complete control and reflection are generalized. For this purpose, a diagnostic component is used, including differentiated tests, exams and questions.

Each of the steps described includes initial, current, and final diagnostics. The purpose of the initial diagnostics is to study the peculiarities of students' readiness to study at the university, the level of formation of motivational components. This information is important for the subsequent organization of the individual educational direction of each student. Current diagnostic tasks include identifying the difficulties faced by students in studying the disciplines, as well as tracking the positive and negative dynamics of learning outcomes for the purpose of subsequent correction. The final diagnostics includes determining the dynamics of the level of formation of the motivational component.

This situation led to a more detailed study of the motivational orientation of engineering students. For this purpose, a diagnostic study was conducted in which it was necessary to determine the level of motivation of engineering students to study natural science disciplines. To do this, we used the diagnostic method and the method "Study of the motivation of learning in higher education".

The study allows us to conclude that there is a high percentage of students with low motivation to study mathematical disciplines.

Students of this group, as a rule, are not interested in studying mathematics. They note that knowledge gained in the audience on these disciplines is unlikely to be useful for them in solving life and professional issues. One of the main reasons for the negative position of students is the high level of abstraction of these disciplines.

The average level of motivation was found in $34.5 \%$ of respondents. Students in this group emphasize the crucial role of mathematics for their future careers and 
recognize the importance of applied course orientation. They actively participated in classes, commented and expressed their position in solving problem situations and used additional literature to perform tasks.

Students with a high level of motivation in the study of mathematical and scientific disciplines (17.2\%) indicate a conscious choice of profession, an interest in studying subjects, a desire for enhanced training and the continuous improvement of their knowledge and skills. Students in this group demonstrated a high level of activity, the pace of work and self-confidence.

The second method is "Studying learning motivation in higher education", which allowed us to draw conclusions about the dominance of such motives as "graduation" and "getting a profession" among students ( $44 \%$ and $37 \%$ respectively). Only $14 \%$ of respondents indicated that the assimilation of knowledge is one of the main reasons for university research.

Thus, analyzing the data and answers of students obtained in the course of diagnostics and conversations in real time, it was found that the reasons for the lack of a high degree of motivation to study mathematics are as follows: a high level of abstraction of educational material; the absence of his "focus" on future professional activities; undeveloped skills of organization and introspection, reflection, work with sources of information, its analysis and systematization.

After the experiment, its implementation in educational practice tasks, we traced the dynamics of the motivation of students in the experimental group in relation to the control.

The study underscores the positive trend towards increasing interest and awareness of students about the importance of mathematical disciplines for future careers: the number of students with a low level decreased from $36.3 \%$ to $18.6 \%$ due to an increase in the number of students with a high level of formation of motivational components. From this study, it follows that the level of motivation of a student is also influenced by his environment.

Thus, the goal of developing education motivation, established at the experiment stage, was successfully implemented by solving differentiated tasks in educational practice.

As a result of this work, we identified factors that ensure the development of learning motivation by solving differentiated tasks:

- motivational-adaptive: taking into account the psycho-physiological characteristics and individual characteristics of students; creating stimulating situations ("success situations", introducing elements of competition, etc.); providing an atmosphere of partnership and dialogical interaction;

- subjective: giving students the right to an independent way of learning with subsequent correction; strengthening of cognitive and educational activities by providing vocational guidance;

- integrative: integration of the content of mathematical and scientific disciplines with elements of professional knowledge based on general concepts and interdisciplinary communication;
- diagnostic and regulatory: the implementation of operational and feedback, thereby ensuring continuous pedagogical support for classroom and outside educational work of students.

Many researchers have studied the problem of developing the motivation of studying students by introducing a tasks system [9, 10]. The study [11] is interesting because they have developed a comprehensive program for the active implementation of practice-oriented tasks. In particular, Mamaev proposes to introduce non-standard tasks and describes a mechanism for the development of student learning motivation and proves the benefits of developing cognitive interest.

In accordance with the logic of our study, the position [12] is the most common. The author notes that the introduction of emotionally significant personal elements into the content of education contributes to the development of intersubjective communication between all participants in the educational process, creates a favorable emotional environment and increases the effectiveness of learning. Educational material is perceived on a personal level if it is perceived as a real task. This, in turn, "mobilizes and, accordingly, develops the structure of the intellect".

The proposed idea of introducing a system of differentiated tasks into educational practices is based on a personally significant approach that allows you to implement the principles of subjectivity, dialogism and individuality.

\section{Conclusion}

The result of the experimental work was the development of student learning motivation through motivational-adaptive (consideration of psychophysiological features and individual characteristics of students, creating stimulating situations (success situations, introducing competition elements, etc.), providing an atmosphere of cooperation and dialogical interaction); a subject (providing students with the right to independently choose the path of study with subsequent correction, strengthening cognitive and learning activities by providing vocational guidance); integration (integration of the content of chemical, mathematical and other scientific disciplines with elements of professional knowledge on the basis of the studied general concepts and interdisciplinary communication); diagnostics and regulation (introduction of operational feedback, ensuring continuous pedagogical support for classroom and outside educational work of students) the capabilities of a system of differentiated tasks.

Perspective directions of this research of problems can be the use of information and communication technologies in teaching disciplines; creation of research projects based on the introduction of interdisciplinary integration of chemical, mathematical and other scientific disciplines.

\section{References}


1. P.P. Blonsky, Selected pedagogical and psychological work (Moscow: Pedagogy, 1979)

2. R.M. Aslanov, Methodical system of differential equations teaching in the pedagogical school of higher education, doctoral thesis (Moscow, 1997)

3. O.V. Bochkareva, Professional orientation in mathematics education for civil engineers in higher education (Saransk, 2006)

4. E.C. Dubovitskaya, Psychological Science and Education, Diagnostic method of learning motivation, 2, 42-46 (2002)

5. A.D. Gladun, Laboratory Workshop on General Physics, vol. 2: Electricity and magnetism (Moscow: MIPT, 2004)

6. G.R. Igtisamova, D.Sh. Nosirov, Peculiarities of problem-solving at studying well drilling with the use of linear differential equations with constant coefficients, Advances in Engineering Research (AER) (Int. Conf. Actual issues of mechanical engineering (AIME 2018)), 157, pp. 211-214 (2018). DOI: 10.2991/aime-18.2018.41

7. E.A. Mukhtasarova, F.G. Safin, State of modern Russian youth tolerance, European Proc. of Social and Behavioural Sci. (RPTSS 2018 - Int. Conf. on res. paradigms transformation in social sci.), 50, pp. 206-213 (2018). DOI: 10.15405/epsbs.2018.12.26

8. K.T. Tyncherov, F.A. Ikhsanova, M.V. Selivanova, Ranking objects by the main component method and highlighting the most informative object parameters, Radioengineering, 9, 185-192 (2018)

9. M.K. Rogachev, V.V. Mukhametshin, Control and regulation of the hydrochloric acid treatment of the bottom-hole zone based on field-geological data, J. of Mining Institute, 231, 275-280 (2018). DOI: 10.25515/PMI.2018.3.275

10. R.T. Akhmetov, V.V. Mukhametshin, Range of application of the Brooks-Corey model for approximation of capillary curves in reservoirs of Western Siberia, Advances in Engineering Res. (AER) (Proc. of the Int. Conf. Actual Issues of Mechanical Engineering (AIME 2018)), 157, pp. 5-8 (2018). DOI: 10.2991/aime-18.2018.2.

11. A.L. Nikitina, Formation of professional competence by the construction and analysis of mathematical models of applied problems, TSU, ser. Humanities, 2, 447-457 (2013)

12. V.V. Serikov, Education and personality. The theory and practice of pedagogical systems' designing (Moscow: Logos) 